\title{
A Case of Acute Myocardial Infarction during Chemotherapy of Advanced Rectal Cancer
}

\author{
Yoshiyuki Kashiwagi ${ }^{1}$ \\ ${ }^{I}$ Graduate School of Health Sciences and Welfare, International University of Health and Welfare, \\ Kanagawa, Japan \\ Corresponding Author: Yoshiyuki Kashiwagi (newstyle.clubana@gmail.com)
}

\begin{abstract}
Background: Cetuximab, irinotecan, levoforinate, and 5-FU (FOLFIRI) are medicines commonly administered to advanced colorectal cancer patients through chemotherapy. Although this regimen is standardized for recurrent metastatic colorectal cancer, the emerging of myocardial infarction is rare.

Purpose: The purpose of this study was to consider the development of myocardial infarction during the chemotherapy of colorectal cancer.

Methods: A retrospective case study was conducted to one patient. An 80-year-old man who developed acute myocardial infarction was in chemotherapy with cetuximab + FOLFIRI with multiple lung metastases after rectal cancer surgery. Data were collected from the patient's medical and nursing records as well as the physiological function test results.

Results: Three days after the 38th administration, the patient visited an emergency outpatient mainly with complaints of dyspnea and back pain. Electrocardiogram showed that the lower wall infarction was suspected. The patient was transferred to a specialized cardiovascular hospital. Emergency coronary angiography was performed in the diagnosis of acute myocardial infarction, and percutaneous coronary intervention was performed. The patient was discharged on the $10^{\text {th }}$ of disease day.

Conclusion: In this case, it was thought that cetuximab + FOLFIRI synergistically induced hyper thrombogenicity, coronary plaque erosion, and acute myocardial infarction. It may also be necessary for interventions such as monitoring the risks in daily living by the medical care providers and guidance on risk avoidance behaviors.
\end{abstract}

Keywords: Acute myocardial infarction; chemotherapy; rectal cancer

\section{BACKGROUND}

Colorectal cancer is the third most common cancer in Japan. Colorectal cancers above stage 3 are recommended to be treated by drip infusion chemotherapy. Some chemotherapy regimens are used; however, most regimens consist of oxaliplatin or irinotecan as the key drug (Endo, Kato, \& Matsui, 2017). Genetic analysis has advanced along the time, therefore drug selection based on genetic analysis results is mainstream for today's clinical practice. RAS gene is one of the key genes for colorectal cancer, and if the patient has wild type RAS gene, the patient can use the molecularly targeted drug (Endo, Kato, \& Matsui, 2017; Komatsu, 2014). Consequently, Cetuximab and irinotecan, levoforinate, 5-FU (FOLFIRI) are administered to advanced colorectal cancer. 
Cetuximab is administered in combination with FOLFIRI for the wild type RAS gene. It is an approved treatment for colorectal cancer in both adjuvant and metastatic cases in Japan (Endo, 2011; Hatake, 2005).

Adverse events of the chemotherapy drugs such as dermal and gastrointestinal toxicity were present in the past, but in rare cases, it was reported that acute myocardial infarction and angina pectoris also emerged during treatment. Among them, many drugs reported to be bevacizumab, cisplatin, 5-FU (Ito \& Mukai, 2018; Komatsu, 2014; Okamoto \& Sasaki, 2015; Takahashi, Tanaka, Tamura, Yamazoe, \& Shibata, 1999; Tsutsumi, Ozawa, Kawakami, Fujii, \& Asamoto, 1990). The onset of cardiovascular disease during chemotherapy is very rare and there are no many cases reported in Japan. However, the onset of cardiovascular disease tends to increase due to the aging of patients and prolonged cancer treatment (Minami, 2010). It is also pointed out that the history of cancer treatment may be a strong risk factor for the onset of heart disease (Armstrong, 2014). In the view of the prognosis of cancer patients and the maintenance of the quality of life, efforts for reducing cardiovascular disease complications are regarded as important (Ito \& Mukai, 2018).

A case of acute myocardial infarction during cetuximab plus FOLFIRI therapy that had rarely been reported in previous studies would be reported through this case report. This report wants to share the development of myocardial infarction during chemotherapy in the clinical practice.

\section{PURPOSE}

The aim of this study was to consider the development of acute myocardial infarction during chemotherapy of colorectal cancer.

\section{METHOD}

A retrospective study of cases in which myocardial infarction developed was conducted. An 80-year-old man who developed acute myocardial infarction was in chemotherapy with cetuximab + FOLFIRI with multiple lung metastases after rectal cancer surgery. Data were collected from the patient's medical and nursing records as well as physiological function test results. The ethical approval was obtained from the target hospital's research ethics committee.

\section{RESULT}

An 80-year old male patient, height $168 \mathrm{~cm}$, weight $53 \mathrm{~kg}$, started chemotherapy with cetuximab plus FOLFIRI (Figure. 1) in April 2016 and obtained a total of 76 doses administered until March 2018. Patient's bloody stools were seen in December 2016; then he visited a hospital. As a result of the examination, a diagnosis of rectal cancer with multiple lung metastases, stage IV was made in January 2016. The patient then had lower anterior colon resection in February 2016. Patient had a past history of hypertension (intrarenal calcium antagonist) with coronary risk factors, including smoking history (40 years up to 18 years ago), drinking history (about two cups daily until 2015), no diagnosis of diabetes but high value of $\mathrm{HbA1C}$ was pointed out (5.3 in April 2016). The patient had a habit of 1-hour walking everyday. 
Table 1. Chemotherapy regimen

\begin{tabular}{llll}
\hline Drugs/day & 1 & 8 & 15 \\
\hline Cetuximab & $\sqrt{ }$ & $\sqrt{ }$ & $\sqrt{ }$ \\
Irinotecan & $\sqrt{ }$ & & $\sqrt{ }$ \\
Levofolinate & $\sqrt{ }$ & & $\sqrt{ }$ \\
Fluorouracil (bolus) & $\sqrt{ }$ & $\sqrt{ }$ \\
Fluorouracil (48hours) & $\sqrt{ }$ & & $\sqrt{ }$ \\
\hline
\end{tabular}

Three days after the 76th administration, dyspnea and back pain occurred during walking in the early morning; then the patient was carried to an emergency department by ambulance. According to the blood biochemical examination (Table 1), a decline in hematopoietic stem function and a high value of white blood cells/neutrophils were observed. Creatine Kinase MB (CK-MB) showed a mild increase of 14.7. Electrocardiogram showed a sinus rhythm with a heart rate of 62 beats per minute with elevated ST segments on II, III, and aVF, and depressed ST segments on V2 - V4. Consequently, the patient was diagnosed with a lower wall acute myocardial infarction, then was transferred to a specialized hospital for the circulatory system.

Table 2. Blood test results

\begin{tabular}{ll}
\hline Components & Value \\
\hline BUN & $21 \mathrm{mg} / \mathrm{dL}$ \\
Creatinine & $0.75 \mathrm{mg} / \mathrm{dL}$ \\
Bilirubine & $0.3 \mathrm{mg} / \mathrm{dL}$ \\
AST & $34 \mathrm{IU} / \mathrm{L}$ \\
ALT & $31 \mathrm{IU} / \mathrm{L}$ \\
LD & $268 \mathrm{U} / \mathrm{L}$ \\
$\gamma$-GT & $42 \mathrm{U} / \mathrm{L}$ \\
CK & $183 \mathrm{U} / \mathrm{L}$ \\
Amylase & $114 \mathrm{U} / \mathrm{L}$ \\
Natrium & $143 \mathrm{mEq} / \mathrm{L}$ \\
Kalium & $3.7 \mathrm{mEq} / \mathrm{L}$ \\
Chloride & $107 \mathrm{mEq} / \mathrm{L}$ \\
Albumin & $4 \mathrm{~g} / \mathrm{dL}$ \\
CK-MB & $14.7 \mathrm{IU} / \mathrm{L}$ \\
CRP & $0.05 \mathrm{mg} / \mathrm{dL}$ \\
WBC & $10400 / \mu \mathrm{L}$ \\
RBC & $365 \times 10^{4} / \mu \mathrm{L}$ \\
Haemoglobin & $11.6 \mathrm{~g} / \mathrm{dL}$ \\
Haematocrit & $35 \%$ \\
Platelets & $15.3 \times 10^{4} / \mu \mathrm{L}$ \\
Neutrophils & $78.9 \%$ \\
HbA1C & 7.1 \\
\hline
\end{tabular}


After trans-placement, an emergency Coronary Angiography (CAG) was performed, finding a complete occlusion of right coronary artery \#2, and $75 \%$ confinement accompanied by strong calcification in \#6-7. For \# 2, PCI percutaneous coronary intervention was performed, and the stent was placed. The patient was discharged from the hospital with good progress after one week of PCI implementation.

\section{DISCUSSION}

There are a number of drugs that have cardiovascular toxicity in chemotherapy drugs (Shirakawa, Kusaba, Odashiro, \& Baba, 2011). Therefore, it might develop acute myocardial infarction during treatment of malignant tumor in daily clinical practice (Suzuki, 2014). Report of cardiovascular events in cetuximab and FOLFIRI used in this case is very few as one case of pulmonary embolism (Goto et al., 2010). However, compared with non-cancer patients, the cardiovascular occurrence is 6.7 times for cancer patients, 28 times for blood tumors, 22.2 times for lung cancer, 20.3 times for gastrointestinal cancer, and for colorectal cancer, it is reported as 16.4 times (Goto et al., 2010).

It is reported that the rate of hospitalization for coronary artery disease within six months of diagnosis of malignant tumor is as high as 1.7 times compared with the non-cancer group. However, in this case, its onset at one year and 11 months, and it decreases as suggested by previous studies (Goto et al., 2010; Suzuki, 2014). Also, the frequency of venous thromboembolism for non-resectable colorectal cancer is generally reported as 08\% (Machials, Sempoux, Scalliet, Coche, \& Humblet, 2007). In chemoradiotherapy using cetuximab, venous thromboembolism is reported to be 3 out of 40 as a serious side effect. As a basis for this, although it is in vivo, inhibition of Epidermal Growth Factor Receptor (EGFR) signaling leads to inhibition of Vascular Endothelial Growth Factor (VEGF) secretion, the relationship between cetuximab and thrombus formation cannot be denied (Pore, Jiang, Gupta, Cerniglia, \& Kao, 2006). Depending on cancer, tissue factor of the coagulation system is expressed abundantly on the cell surface, and various cytokines such as angiogenic factors are produced. In the process, it is said to be in a hypercoagulable state, which is said to be prone to atherosclerotic artery thrombosis (Ito \& Mukai, 2018).

Besides drug thrombus formation, the relationship with conventionally said dangerous risk factors in everyday life was also considered. Compared with chemotherapy, the patient had an increase of about 10 kilograms in weight during chemotherapy before the onset of myocardial infarction. Moreover, high levels of HbA1C were also seen after chemotherapy. Generally, the patient who used steroid might be having impaired glucose tolerance (IGT) (Takano, 2017). As a factor related to high levels of HbA1C, druginduced glucose tolerance abnormality due to the use of steroid which is a premedication for chemotherapy may be considered, but an overdose of sugar was routinely observed. Because it was palliative chemotherapy, even though grasping such lifestyle habits did not make teaching so severe, it is considered to be a factor causing cardiovascular events. However, in the case where a long-term response state is observed as in this case, factors such as lifestyle such as diet and exercise and aging may combine to develop cardiovascular disease (Ito \& Mukai, 2018). 
Due to the improvement of antiemetic drugs, there is an individual difference, but the risk of nausea and vomiting is extremely decreased, so the idea that chemotherapy with nausea and malaise decreases meal volume is considered to be in the past. There are no nausea like in this case, and there are patients who eat various things freely. Therefore, ordinary things are needed to be instructed e.g. that it is not the idea that "patients can eat anything any number" by palliative chemotherapy, but to eat proper amounts and maintain appropriate weights (Komatsu, 2014).

When surgery is performed for colon cancer and overcoming the acute phase, it is possible to improve the prognosis for 36 months by performing chemotherapy. Although this will result in death after 36 months, patients will enter a chronic phase of living with cancer. Perhaps, a paradigm shift is needed for the guideline that our cancer patients follow. It is easy to think that myocardial infarction-like this case develops due to complex factors of chemotherapy, malignant tumor, and daily life habits (Ito \& Mukai, 2018; Minami, 2010).

Through this case, it is important to conduct daily living guidance and monitoring for the prevention of cardiac diseases and to make efforts to continue safe chemotherapy. To that end, it is thought that it is necessary to identify and intervene the risk factors that are present or latent in patients through the clinical team such as nurses, doctors, pharmacists, and administrative nutritionists.

\section{CONCLUSION}

In this case, it is thought that cetuximab + FOLFIRI synergistically induced hyper thrombogenicity, coronary plaque erosion, and acute myocardial infarction. It may also be necessary for interventions such as monitoring of risks in daily living by the medical care provider and guidance on risk avoidance behaviors.

\section{REFERENCES}

Armstrong, G. (2014). Aging and risk of severe, disabling, life-threatening, and fatal events in the childhood cancer survivor study. J Clin Oncol, 1218-1227.

Elder, R., Neal, C., Davis, B. A., Almes, E., Whitledge, L., \& Littlepage, N. (2004). Patient Satisfaction with triage nursing in a rural hospital emergency department. Journal of Nursing Care Quality, 19, 265-268.

Endo, K., Kato, H., \& Matsui, R. (2017). Treatment regimens for cancer chemotherapy. Tokyo: Yodosha.

Goto, A., Suzuki, K., Hasegawa, Y., Sukawa, Y., Fujii, K., \& Nishimura, S. (2010). Pulmonary embolism during palliative chemotherapy including cetuximab for metastatic colorectal cancer. Jpn J Cancer Chemother, 37(1), 169-171.

Ito, H., \& Mukai, M. (2018). Onco-cardiology. Tokyo: Bunko-sha.

Komatsu, Y. (2014). Professional management techniques of gastrointestinal cancers chemotherapy side effect. Tokyo: Medical View.

Laschinger, H. S., Hall, L. M., Pedersen, C., \& Almost J. (2005). A psychometric analysis of the patient satisfaction with nursing care quality questionnaire: an actionable approach to measuring patient satisfaction. Journal of Nursing Care Quality, 20(3), $220-230$. 
Machials, J. P., Sempoux, C., Scalliet, P., Coche, J. C., \& Humblet, Y. (2007). Phase I/II study of preoperative cetuximab, capecitabine, and external beam radiotherapy in patients with rectal cancer. Ann Oncol, 18(4), 738-744.

Minami, M. (2010). Cardiovascular side-effects of modern cancer therapy. Circ J, 17791786.

Okamoto, R., \& Sasaki, T. (2015). Handbook of Cancer Chemotherapy Side Effects: The Treatment and Prevention Second Edition. Tokyo: Yodosha.

Pore, N., Jiang, Z., Gupta, A., Cerniglia, G., \& Kao, G. D. (2006). EGFR tyrosine kinase inhibitors decrease VEGF expression by both hypoxia-inducible factor (HIF)-1independent and HIF-1-dependent mechanisms. Cancer Res, 66(6), 3197-3204.

Shattell, M. (2004). Nurse-patient interaction: A review of literature. Journal of Clinical Nursing, 13, 714 - 722 .

Shirakawa, T., Kusaba, H., Odashiro, K., \& Baba, E. (2011). Retrospective study of 8 patients who developed venous thromboembolism during cancer chemotherapy. HEART, 43(7), 1006-1009.

Suzuki, H. (2014). Editorial Comment to Okada's study. HEART, 46(4), 517-518.

Takahashi, M., Tanaka, Y., Tamura, Y., Yamazoe, M., \& Shibata, A. (1999). A case report of simulating acute myocardial infarction associated with cardiotoxicity of anticancer agents. Clinic All-Round, 48(1), 193-196.

Tsutsumi, T., Ozawa, Y., Kawakami, A., Fujii, H., \& Asamoto, H. (1990). Acute myocardial infarction induced by lung cancer chemotherapy with cisplatin and etoposide. Jpn J Cancer Chemother, 17(3), 413-417. 